\title{
Ângulo de fase padronizado e sua relação com parâmetros antropométricos e inflamatórios em mulheres com lúpus eritematoso sistêmico
}

\author{
Standardized phase angle and its relationship to anthropometric and inflammatory parameters \\ in women with systemic lupus erythematosus
}

\section{DOI: $10.37111 /$ braspenj.2019344003}

Ana Lina de Carvalho Cunha Sales ${ }^{1}$

Elieide Soares de Oliveira ${ }^{2}$

Maísa Guimarães Silva Primo ${ }^{3}$

Thamara Martins Silva ${ }^{4}$

Islanne Leal Mendes ${ }^{4}$

Gilmara Péres Rodrigues 5

Maria do Carmo de Carvalho e Martins ${ }^{5}$

\section{Unitermos:}

Lúpus eritematoso sistêmico. Inflamação. Antropometria.

\section{Keywords:}

Lupus erythematosus, systemic. Inflammation. Anthropometry.

\section{Endereço para correspondência:}

Ana Lina de Carvalho Cunha Sales

Campus Universitário Ministro Petrônio Portela, SG 07

$\mathrm{s} / \mathrm{n}$ - Ininga - Teresina, PI, Brasil - CEP: 64049-550

E-mail: ana.lina123@gmail.com

\section{Submissão}

28 de agosto de 2019

Aceito para publicação

20 de outubro de 2019

\section{RESUMO}

Introdução: O ângulo de fase está associado a alterações no estado nutricional, podendo ser utilizado para o diagnóstico nutricional, bem como indicador de prognóstico ou preditor de sobrevida em algumas situações clínicas. $\mathrm{O}$ objetivo deste estudo foi relacionar o ângulo de fase padronizado (AFP) com parâmetros antropométricos e inflamatórios, em mulheres com lúpus eritematoso sistêmico (LES). Método: Trata-se de estudo transversal, realizado com 23 mulheres diagnosticadas com LES. Avaliaram-se peso $(\mathrm{kg})$ e altura $(\mathrm{m})$, para determinação do índice de massa corporal (IMC), e aferiu-se a circunferência da cintura $(C C)$, conforme normas vigentes. $\mathrm{O}$ valor de hemossedimentação (VHS) e proteína $\mathrm{C}$ reativa (PCR) foram obtidos nos prontuários médicos. Obteve-se o AFP por Bioimpedância Elétrica Segmentar Direta Multifrequência, Inbody S-10, corrigindo-se o valor obtido pelo desvio padrão, de acordo com idade e sexo. A análise estatística incluiu a análise da simetria de distribuição dos dados por Shapiro-Wilk e a aplicação de testes de correlação adequados aos tipos de variáveis avaliadas, considerando significativos valores de $p<0,05$. Resultados: A média de idade dos pacientes foi de $34,5 \pm 11,4$ anos, com valor médio do IMC $\left(27,7 \pm 3,7 \mathrm{~kg} / \mathrm{m}^{2}\right)$ compatível com excesso de peso corporal. A média de CC $(88,5 \pm 10,8 \mathrm{~cm})$ indicou adiposidade abdominal e elevado risco cardiometabólico. Em média, o $\operatorname{AFP}(-1,23 \pm 0,7)$ das mulheres indicou risco nutricional, caracterizado por menor quantidade de massa muscular e elevado \% de gordura corporal (36\%). Valores elevados de VHS e PCR também foram encontrados na população estudada. Não foi verificada correlação entre AFP, parâmetros antropométricos e inflamatórios nas mulheres avaliadas $(p>0,05)$. Conclusão: As mulheres com LES estão em risco nutricional, conforme indicado pelo AFP, sem relação com os parâmetros antropométricos e inflamatórios avaliados.

\section{ABSTRACT}

Introduction: The phase angle is associated with changes in nutritional status and could be used for nutritional diagnosis, as well as prognostic indicator or survival predictor in some clinical situations. The aim of this study was to associate the standardized phase angle (AFP) to anthropometric and inflammatory parameters in women with systemic lupus erythematosus (SLE). Methods: A cross-sectional study conducted with 23 women diagnosed with SLE. Weight $(\mathrm{kg})$ and height $(\mathrm{m})$ were evaluated to determine body mass index (BMI), and waist circumference (WC) was measured according to current standards. Erythrocyte sedimentation (ESR) and C-reactive protein (CRP) values were obtained from medical records. AFP was obtained by Direct Segmental Multi-Frequency Electrical Bioimpedance, Inbody S-10, and the value obtained by the standard deviation was corrected according to age and gender. Statistical analysis included Shapiro-Wilk's analysis for symmetry of data distribution and the application of appropriate correlation tests to the types of variables evaluated, considering significant values of $p<0.05$. Results: The mean age of the patients was $34.5 \pm 11.4$ years, with a mean BMl $\left(27.7 \pm 3.7 \mathrm{~kg} / \mathrm{m}^{2}\right)$ compatible with excess body weight. The WC mean $(88.5 \pm 10.8 \mathrm{~cm})$ indicates abdominal adiposity and high cardiometabolic risk. On average, women's AFP (-1.23 \pm 0.7$)$ indicates nutritional risk, characterized by less muscle mass and a higher \% of body fat (36\%). High ESR and CRP values were also found in the study population. No correlation was found between AFP, anthropometric and inflammatory parameters in the women evaluated ( $p>0.05$ ). Conclusion: Women with SLE are at nutritional risk, as indicated by AFP, unrelated to the anthropometric and inflammatory parameters evaluated.

1. Doutoranda, Nutricionista do Hospital Universitário da Universidade Federal do Piauí, Teresina, Pl, Brasil.

2. Especialista em Alta Complexidade, Residência pelo Instituto de Saúde Coletiva da Universidade Federal da Bahia, Salvador, BA, Brasil.

3. Especialista em Alta Complexidade, Nutricionista Mestranda do Programa de Pós-Graduação em Alimentos e Nutrição da Universidade Federal do Piauí, Teresina, PI, Brasil.

4. Mestre, Nutricionista da Universidade Federal do Piauí, Teresina, Pl, Brasil.

5. Doutora, Nutricionista e Professora da Universidade Federal do Piauí, Teresina, PI, Brasil. 


\section{INTRODUÇÃO}

O lúpus eritematoso sistêmico (LES) é uma doença inflamatória de caráter autoimune, de etiologia multifatorial, envolvendo aspectos genéticos, ambientais, hormonais, infecciosos e psicológicos ${ }^{1}$. As manifestações clínicas mais comuns incluem lesões na pele e inflamação nas articulações, podendo acometer órgãos, como rins e membranas serosas, além de alterar as células sanguíneas.

De maior predominância na população feminina em idade reprodutiva (20 a 40 anos), não há números exatos da incidência e prevalência do LES no Brasil. Mas, segundo a Sociedade Brasileira de Reumatologia, as estimativas indicam que 65.000 pessoas têm LES, correspondendo a uma em cada 1.700 mulheres brasileiras².

Pacientes com LES têm risco aumentado de desenvolver comorbidades, como dislipidemia, diabetes mellitus, doenças cardiovasculares, obesidade e síndrome metabólica, decorrente da própria patogênese da doença ${ }^{3}$. Assim, o tratamento de LES irá depender da gravidade da doença e de suas complicações.

As abordagens de terapia não-farmacológica incluem suporte psicossocial, atividade física e nutricional. Baseado nisso, o estado nutricional é de suma importância para manter o equilibro orgânico e evitar quadros associados a infecções, complicações metabólicas, morbimortalidade e aumento dos custos hospitalares ${ }^{4}$.

A avaliação do estado nutricional ainda não dispõe de um padrão para o acompanhamento de alterações nutricionais com nível de sensibilidade e eficácia. Na ausência de um único método, é recomendada a combinação de parâmetros antropométricos, composição corporal e laboratoriais ${ }^{5}$. Uma alternativa é a utilização do ângulo de fase $(\mathrm{AF})$, um parâmetro relativamente novo, obtido por meio da bioimpedância elétrica (BIA), capaz de avaliar a integridade celular e a distribuição hídrica, pela medida direta entre reactância e resistência de tecidos corporais. Dessa forma, quanto mais íntegras estiverem as membranas, maior será o armazenamento de energia e o $\mathrm{AF}^{6}$.

Tem-se verificado um interesse por estudar o AF, por este ser um método objetivo, prático e não-invasivo, considerado como bom indicador de estado de saúde e ferramenta de diagnóstico nutricional em diversas doenças ${ }^{7}$. Estudos são escassos quanto à aplicação desse parâmetro em pacientes com LES, assim, o objetivo desse estudo foi avaliar a existência de relação do $\mathrm{AF}$ com os parâmetros antropométricos e inflamatórios em mulheres com LES.

\section{MÉTODO}

Trata-se de um estudo transversal, descritivo e observacional, realizado em mulheres com LES atendidas no ambulatório de Reumatologia de um Hospital Universitário do Nordeste do Brasil. Foram incluídas mulheres com idade entre 18 e 59 anos, com diagnóstico de LES segundo os critérios do American College of Rheumatology (ACR).

A amostra foi determinada por metodologia não-probabilística de conveniência. A coleta de dados foi realizada nos meses de agosto a outubro de 2018 , totalizando 3 meses.

O índice de massa corporal (IMC) foi obtido após aferição do peso corporal $(\mathrm{kg})$ e estatura $(\mathrm{m})$, utilizando-se balança digital portátil, com capacidade máxima de $150 \mathrm{~kg}$ e precisão de $100 \mathrm{~g}$, bem como antropômetro graduado em centímetros, em barra vertical fixa para posicionamento sobre a cabeça. A classificação do estado nutricional foi realizada segundo os pontos de corte do IMC $\left(\mathrm{kg} / \mathrm{m}^{2}\right)$, propostos pela Organização Mundial de Saúde (OMS) ${ }^{8}$.

Para aferição da circunferência da cintura (CC) foi utilizada fita métrica flexível e inelástica, com precisão de 0,1 $\mathrm{cm}$, posicionada circundando a linha natural da cintura, no ponto médio entre a última costela e a crista ilíaca. A classificação da adiposidade abdominal e o risco cardiometabólico baseou-se nos valores de referência da OMS?.

A composição corporal foi avaliada com auxílio de Bioimpedância Elétrica Segmentar Direta Multifrequência (DSM-BIA) s 10 Inbody ${ }^{\circledR}$, portátil, tetrapolar, com oito eletrodos, conforme protocolo de jejum estabelecido pelo fabricante ${ }^{10}$. Os dados relativos à composição corporal foram utilizados para determinação do $A F$ e ângulo de fase padronizado (AFP), utilizando-se fórmula proposta por Barbosa-Silva et al. ${ }^{6}$, que considera a correção pelo desvio padrão, idade e sexo. Os valores abaixo de - 1,65 (representa o percentil 5) foram classificados como indicadores de risco nutricional.

Os marcadores inflamatórios utilizados neste estudo foram o valor de hemossedimentação (VHS) e proteína C reativa (PCR), complementos $\mathrm{C} 3$ e C4. Os dados bioquímicos foram obtidos por meio de consulta aos prontuários médicos e classificados conforme valores de referência do laboratório de análises clínicas do Hospital Universitário da Universidade Federal do Piauí (HU-UFPI).

Os dados foram organizados em planilha do Excel e, posteriormente, exportados ao software Statistical Package for Social Sciences (SPSS, versão 22.0) para análise estatística. Foi aplicado o teste Shapiro-Wilk para verificar a normalidade da distribuição dos dados. Em seguida, aplicaram-se os testes de correlação de Pearson e Spearman, para variáveis contínuas paramétricas e não-paramétricas, respectivamente. Adotou-se significância de 5\%, com índice de confiança de $95 \%$.

Este estudo seguiu as recomendações da Resolução $n^{\circ}$ 466/2012 do Conselho Nacional de Saúde (CNS), com obtenção do consentimento formal escrito de todas as 
participantes, e foi submetido à Comissão de Avaliação de Projetos de Pesquisa (CAPP) e Comitê de Ética em Pesquisa do HU-UFPI, com aprovação sob CAAE $n^{\circ}$ : 880971 18.9.0000.8050.

\section{RESULTADOS}

Foram recrutadas 45 pessoas, das quais 22 foram excluídas, sendo 9 por apresentarem lesão ou rigidez de membros que impossibilitaram o posicionamento na bioimpedância, 4 por processo patológico infeccioso e 8 por não retornarem para avaliação da composição corporal. Ao final, a amostra foi constituída por 23 mulheres diagnosticadas com LES.

A Tabela 1 demonstra as características demográficas das pacientes com LES, verificando-se que a maioria possuía etnia parda $(65,2 \%)$, com grau de escolaridade equivalente ao ensino médio completo (78,2\%). A média de idade das mulheres avaliadas foi de 34,5 $\pm 11,4$ anos.

Quanto à avaliação antropométrica, os resultados demonstraram excesso de peso pelo IMC $(82,6 \%)$ e adiposidade abdominal pela CC $(52,2 \%)$, indicativa de elevado risco cardiometabólico. A avaliação da composição corporal mostrou percentuais elevados de gordura corporal e risco nutricional em $56,5 \%$ das mulheres, segundo indicado pelo AFP (Tabela 2).

Tabela 1 - Características demográficas de mulheres com lúpus eritematoso sistêmico.

\begin{tabular}{lcc}
\hline Variáveis & N & $\%$ \\
\hline Etnia & 7 & \\
Preta & 15 & 60,4 \\
Pardo & 1 & 4,3 \\
Branco & & \\
\hline Escolaridade & 3 & 13,0 \\
Ensino Fundamental & 18 & 78,2 \\
Ensino Médio & 2 & 8,7 \\
Ensino Superior & \\
\hline
\end{tabular}

Tabela 2-Valores médios e desvios padrão da idade, parâmetros antropométricos, composição corporal e ângulo de fase padronizado de mulheres com lúpus eritematoso sistêmico.

\begin{tabular}{lc}
\hline Parâmetro & Média $\pm \mathrm{DP}$ \\
\hline Idade $($ anos $)$ & $34,5 \pm 11,4$ \\
IMC $\left(\mathrm{kg} / \mathrm{m}^{2}\right)$ & $27,7 \pm 3,7$ \\
$\mathrm{CC}(\mathrm{cm})$ & $88,5 \pm 10,8$ \\
$\mathrm{GC}(\%)$ & $36,2 \pm 5,3$ \\
MME $(\mathrm{kg})$ & $23,7 \pm 5,1$ \\
AFP $(\mathrm{Xc} / \mathrm{R})$ & $-1,23 \pm 0,7$ \\
\hline
\end{tabular}

IMC = Índice de massa corporal; $\mathrm{CC}=$ circunferência da cintura; $\mathrm{GC}=$ gordura corporal; $\mathrm{MME}=$ massa muscular esquelética; AFP = ângulo de fase padronizado.
Com relação aos marcadores inflamatórios, observou-se valores elevados de PCR e VHS (Tabela 3).

A Tabela 4 demonstra os resultados da análise de correlação entre AFP e parâmetros antropométricos, composição corporal e marcadores inflamatórios, verificando-se que não houve valores estatisticamente significativos $(p>0,05)$ entre as variáveis analisadas.

Tabela 3 - Valores médios e desvios padrão dos marcadores inflamatórios avaliados de mulheres com lúpus eritematoso sistêmico.

\begin{tabular}{ll}
\hline Marcadores & Média \pm DP \\
\hline PCR & $25,2 \pm 54,9$ \\
VHS & $40,9 \pm 32,1$ \\
\hline
\end{tabular}

PCR = proteína C reativa; VHS = valor de hemossedimentação.

Tabela 4 - Coeficientes de correlação entre ângulo de fase padronizado e variáveis antropométricas e inflamatórias de mulheres com lúpus eritematoso sistêmico.

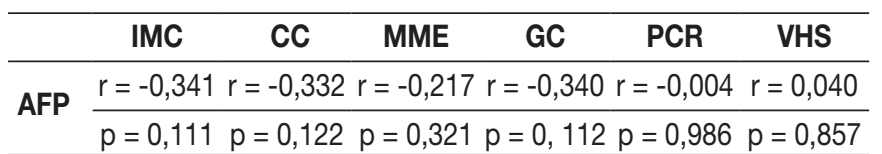

*valores significativos para Pearson ou Spearman; AFP = ângulo de fase padronizado; IMC = índice de massa corporal; $\mathrm{CC}=$ circunferência da cintura; $\mathrm{GC}=$ gordura corporal; $\mathrm{MME}=$ massa muscular esquelética; $\mathrm{PCR}$ = proteína $\mathrm{C}$ reativa; $\mathrm{VHS}$ = valor de hemossedimentação.

\section{DISCUSSÃO}

No presente estudo, durante o período de coleta, apenas um homem constou nas consultas, sendo excluído pela impossibilidade de realizar dados estatísticos, caracterizando uma amostra de mulheres. Marnet e Gonçalves ${ }^{11}$ afirmam que LES é 10 vezes mais prevalente em mulheres do que em homens e isso pode ser justificado pela ação dos hormônios femininos, como estrogênio, favorecendo o enfraquecimento dos linfócitos $T$ supressores e fortalecendo a resposta dos linfócitos T auxiliares.

A média de idade desta população foi de $34,5 \pm 11,4$ anos, estando de acordo com a literatura, que situa a manifestação da doença em mulheres em idade fértil (15 a 45 anos) $)^{12,13}$. Achados semelhantes foram obtidos por Nakashima et al. ${ }^{14}$, em investigação sobre a incidência e aspectos clínico-laboratoriais do LES em cidade do Sul do Brasil, que identificou que o pico de incidência da doença aconteceu na faixa etária de 30 a 39 anos.

Com relação ao nível de escolaridade dos pesquisados, percebeu-se que a maioria concluiu o ensino médio $(78,2 \%)$. Em estudo realizado por Tavares et al. ${ }^{15}$, sobre baixa adesão ao tratamento de doenças crônicas no Brasil, notou-se que indivíduos com menor escolaridade tinham maior dificuldade de concordância com o tratamento e um bom nível de 
escolaridade contribui para a compreensão das orientações fornecidas pela equipe multiprofissional, bem como na aderência à terapia medicamentosa.

Quando avaliado o estado nutricional considerado pelo IMC e a adiposidade corporal segundo a CC, observou-se excesso de peso, confirmado pelo aumento do percentual de gordura corporal, além de risco cardiometabólico, por valores acima do recomendado. Esses dados corroboram os achados de Almeida et al. ${ }^{4}$, em estudo realizado com 40 mulheres portadores de LES, em que $40 \%$ apresentavam excesso de peso corporal e, em 90\%, a CC tinha valores elevados.

O excesso de peso e de gordura na região abdominal traz risco para o desenvolvimento de outras doenças, como síndrome metabólica, pois o tecido adiposo branco é capaz de ativar uma cascata inflamatória com a produção principalmente de fator de necrose tumoral alfa (TNF) e interleucina 6 (IL-6). Além disso, o aumento dos ácidos graxos livre por um metabolismo de lipídios alterado contribui para a infiltração de células imunológicas, como os macrófagos, aumento da produção de citocinas inflamatórias, que por sua vez causam uma disfunção no metabolismo da insulina $^{16}$.

Cabe ressaltar que o tratamento com glicocorticoides, fármacos mais comuns para o tratamento de LES, contribui para o acúmulo de gordura, e suas doses diárias irão diferir de acordo com protocolo individualizado. De acordo com pesquisa realizada por Mok et al. ${ }^{17}$, que avaliaram 29 pacientes com LES por seis meses, os pacientes que receberam doses elevadas de corticoides tinham correlação com mudanças no IMC, elevação do percentual de gordura e redução de massa magra.

Observa-se o papel da alimentação como agente modulador da inflamação para diminuir os riscos de doenças cardiovasculares, visto que são doenças comuns em pacientes com LES. Pocovi-Gerardino et al.' investigaram a relação do índice inflamatório da dieta com marcadores de doenças cardiovasculares em mulheres com LES e verificaram que o maior potencial inflamatório da dieta estava associado a níveis elevados de colesterol total. Ressalta-se que nessa pesquisa não foi possível realizar avaliação do consumo alimentar, impossibilitando comparações com marcadores do risco de doenças cardiovasculares.

Prado et al. ${ }^{18}$ demonstraram que $40 \%$ dos pacientes com LES estudados ( $n=40)$ apresentaram parâmetros inflamatórios elevados e os autores associaram com a baixa eficácia da terapia instituída. Achados semelhantes foram encontrados no presente estudo, com identificação de valores aumentados para PCR e VHS. Por outro lado, fatores como dieta inadequada, sedentarismo e obesidade também contribuem para a inflamação.
Quando avaliado o AFP, verificou-se risco de alterações nas membranas, podendo ser explicado pela menor massa muscular entre mulheres e elevado porcentual de gordura corporal. Quando correlacionados AFP com parâmetros antropométricos e marcadores inflamatórios, não houve diferenças estatisticamente significativas $(p>0,05)$ e esses resultados podem estar relacionado ao reduzido tamanho amostral.

Na pesquisa de Costa ${ }^{19}$, verificou-se que havia correlação positiva e moderada com valores estatisticamente significativos ( $p<0,001$ ) entre AFP e IMC. Isso pode ser explicado pelo fato do AF estar diretamente relacionado a membranas celulares, o que levaria a crer que esses valores são diretamente proporcionais.

Em contrapartida, Carvalho et al. ${ }^{20}$ demonstraram que, em pacientes hospitalizados, o IMC não apresentou valores significativos quando relacionado com o AFP. No entanto, a circunferência do braço apresentou uma correlação positiva de fraca a moderada intensidade. Os autores analisaram, ainda, a concordância dessas variáveis e identificaram baixa concordância, o que poderia ser explicado pelo fato do AFP e parâmetros antropométricos expressarem diferentes níveis de deficiência nutricional.

Não foram encontrados estudos na literatura que tenham avaliado AFP em pacientes com LES, o que pode ser o passo inicial de uma nova ferramenta para a busca de estratégias nutricionais mais assertivas para essas pacientes.

\section{CONCLUSÃO}

Conclui-se que as mulheres portadoras de LES desse estudo estavam em período reprodutivo e apresentavam excesso de peso, gordura corporal e adiposidade abdominal acima dos valores recomendados, indicando risco de desenvolver outras comorbidades. Salienta-se que o AFP revelou risco, mas não teve valores significativos quando correlacionados com parâmetros antropométricos (IMC e $\mathrm{CC}$ ) e inflamatórios (PCR e VHS).

Sugere-se que a relação entre AF e parâmetros antropométricos e inflamatórios precisa ser mais bem esclarecida, sendo necessários estudos que incluam maior número de pacientes, diferentes fases da doença e apresentações clínicas distintas, bem como diferentes fases do tratamento, considerando o uso de drogas farmacológicas.

\section{REFERÊNCIAS}

1. Pocovi-Gerardino G, Correa-Rodriguez M, Callejas-Rubio JL, Rios-Fernandes R, Martín-Amada M, Cruz-Caparros MG, et al. Dietary inflammatory index score and cardiovascular disease risk markers in women with systemic lupus erythematosus. J Acad Nutr Diet. 2019. pii: S2212-2672(18)32082-3. 
2. Sociedade Brasileira de Reumatologia - Comissão de Lúpus. Cartilha de reumatologia. [cited 2019 Oct 10]. Disponível em: https://www.reumatologia.org.br/doencas-reumaticas/lupuseritematoso-sistemico-les/ .

3. Costi LR, Iwamoto HM, Neves DCO, Caldas CAM. Mortalidade por lúpus eritematoso sistêmico no Brasil: avaliação das causas de acordo com o banco de dados de saúde do governo. Rev Bras Reumatol. 2017;57(6):574-82.

4. Almeida CS, Mendes ALRF, Cavalcante ACM, Arruda SPM, Silva FR, Albuquerque LP. Perfil antropométrico e consumo alimentar de mulheres com lúpus eritematoso sistêmico. Arq Catarin Med. 2017;46(4):103-17.

5. Silva DMM, Santos CM, Moreira MA. Perfil nutricional de pacientes internados em um hospital público de Recife- PE. Dest Acad (Lajeado). 2016;8(3):97-108.

6. Barbosa-Silva MC, Barros AJ, Wang J, Heymsfield SB, Pierson RN Jr. Bioelectrical impedance analysis: population reference values for phase angle by age and sex. Am J Clin Nutr. 2005;82(1):49-52.

7. Paiva SI, Borges LR, Halpern-Silveira D, Assunção MC, Barros AJ, Gonzalez MC. Standardized phase angle from bioelectrical impedance analysis as prognostic factor for survival in patients with cancer. Support Care Cancer. 2010;19(2):187-92.

8. World Health Organization. Technical report series - physical status: the use and interpretation of antropometry. Geneva: World Health Organization; 2000.

9. World Health Organization. Waist circumference and waist-hip ratio: report of a WHO expert consultation. Geneva: World Health Organization; 2005.

10. Rossi L, Caruso L, Galante AP. Avaliação nutricional: novas perspectivas. São Paulo: Roca; 2008.

11. Marnet EBO, Gonçalves M. Lúpus eritematoso sistêmico e sua associação com doenças psiquiátricas: revisão da literatura.
Psychiatry on line Brasil. 2016;21(7). [cited 2019 Sep 3]. Disponível em: https://www.polbr.med.br/ano16/prat0716.php

12. Rossoni C, Keiserman MW, Staub HL. Perfil nutricional e metabólico de pacientes com lupus eritematoso sistêmico de um centro de referência. Rev Bras Nutr Clin. 2010;25(2):151-7.

13. Costa LM, Coimbra CCBE. Lúpus eritematoso sistêmico: incidência e tratamento em mulheres. Uningá Review. 2014;20(1):81-6.

14. Nakashima CAK, Galhardo AP, Silva JFM, Fiorenzano GR, Santos ABS, Leite MFS, et al. Incidência e aspectos clínicolaboratoriais do lúpus eritematoso sistêmico em cidade do Sul do Brasil. Rev Bras Reumatol. 2011;51(3):231-9.

15. Tavares NUL, Bertoldi AD, Mengue SS, Arrais PSD, Luiza VL, Oliveira MA, et al. Fatores associados à baixa adesão ao tratamento farmacológico de doenças crônicas no Brasil. Rev Saúde Pública. 2016;50(suppl. 2):10s.

16. Torres S, Fabersani E, Marquez A, Gauffin-Cano P. Adipose tissue inflammation and metabolic syndrome. The proactive role of probiotics. Eur J Nutr. 2019;58(1):27-43.

17. Mok CC, To CH, Ma KM. Changes in body composition after glucocorticoid therapy in patients with systemic lupus erythematosus. Lupus 2008;17(11):1018-22.

18. Prado DM, Amaral BA, Duarte SFP, Vale AE, Silva ML, Rocha AR, et al. Perfil dos pacientes portadores de lúpus eritematoso sistêmico atendidos pelo componente especializado da assistência farmacêutica em uma regional de saúde. Rev Mult Psic. 2017;11(38):808-23.

19. Costa GLOB. Ângulo de fase nutricional enquanto indicador no câncer do trato digestório [Dissertação de Mestrado]. Salvador: Universidade Federal da Bahia; 2012.

20. Carvalho JAS, Dias TMS, Primo MGS, Aguiar JRS, Moura e Silva MC, Carvalho LR, et al. Ângulo de fase em pacientes hospitalizados: relação com parâmetros antropométricos. BRASPEN J. 2018;33(3):303-7.

Local de realização do estudo: Hospital Universitário da Universidade Federal do Piauí, Teresina, PI, Brasil.

Conflito de interesse: As autoras declaram não haver.

Foi apresentado como trabalho de destaque no XXIII Congresso Brasileiro de Nutrição Parenteral e Enteral, entre 20 a 23 de outubro de 2019, em Foz do Iguaçu-PR. 